\title{
A Theorem of Sonine in Bessel Functions, with two Extensions to Spherical Harmonics.
}

\author{
By Dr Johy Dougall.
}

(Read 18th December 1918. Received 15th March 1919.)

1. In his notable memoir on the Bessel Functions,* Sonine proves the elegant result that

$$
\begin{gathered}
\int_{0}^{\infty} J_{m}(\lambda a) J_{m}(\lambda b) J_{m}(\lambda c) \lambda^{-m+1} d \lambda \\
=\frac{\{(a+b+c)(b+c-a)(c+a-b)(a+b-c)\}^{m-1}}{\sqrt{\pi} 2^{3 m-1} \Pi\left(m-\frac{1}{2}\right) a^{m} b^{m} c^{m}},
\end{gathered}
$$

$\left(m>-\frac{1}{2}\right)$, provided a plane triangle can be drawn with sides $a, b, c$; otherwise the value of the integral is zero.

The present paper contains-

(i) a proof of Sonine's Theorem based immediately on the Theory of the Potential;

(ii) a theorem in Spherical Harmonics, with proof precisely analogous to the proof (i);

(iii) statement of a second theorem in Spherical Harmonics, totally unlike the theorem (ii), except in this that both theorems contain Sonine's result as a limiting case.

The two theorems in Spherical Harmonics areFirst,

$$
\begin{aligned}
& \sum_{p=0}^{p=\infty}(2 m+2 p+1) \frac{\Pi(2 m+p)}{p !} P_{m+p}^{m}(\cos \alpha) P_{m+p}^{m}(\cos \beta) P_{m+p}^{m}(\cos \gamma) \\
= & \frac{\left(\sin \frac{\alpha+\beta+\gamma}{2} \sin \frac{\beta+\gamma-\alpha}{2} \sin \frac{\gamma+\alpha-\beta}{2} \sin \frac{\alpha+\beta-\gamma}{2}\right)^{m-\frac{1}{2}}}{\sqrt{\pi} \Pi\left(m-\frac{1}{2}\right) 2^{-m} \sin ^{m} \alpha \sin ^{m} \beta \sin ^{m} \gamma}, \ldots \ldots
\end{aligned}
$$

$\left(m>-\frac{1}{2}\right)$, provided a spherical triangle can be drawn with sides $\alpha, \beta, \gamma$; otherwise the value of the series is zero;

- Math. Annalen, Band xvi. (1880). 
Second,

$$
\int_{0}^{1}\left(1-\mu^{2}\right)^{-\frac{m}{2}} P_{m+n}^{m}(\mu) P_{m+p}^{m}(\mu) P_{m+q}^{m}(\mu) d \mu
$$

$=\frac{2^{m-1} \Pi n \Pi p \Pi q \Pi\left(2 m+\frac{n+p+q}{2}\right) \Pi\left(m-\frac{1}{2}+\frac{p+q-n}{2}\right) \Pi\left(m-\frac{1}{2}+\frac{q+n-p}{2}\right) \Pi\left(m-\frac{1}{2}+\frac{n+p-q}{2}\right)}{\sqrt{ } \bar{\pi} \Pi\left(m-\frac{1}{2}\right) \Pi\left(m+\frac{1}{2}+\frac{n+p+q}{2}\right) \Pi(2 m+n) \Pi(2 m+p) \Pi(2 m+q) \Pi \frac{p+q-n}{2} \Pi \frac{q+n-p}{2} \Pi \frac{n+p-q}{2}}$

( $m>-1$ ), where $n, p, q$ are positive integers whose sum is even. If one of the three integers $n, p, q$ is greater than the sum of the other two, the value of the integral is zero, as the formula itself shows.

The definition taken for $P_{t}^{m}(\mu)$ is *

$$
P_{t}^{m}(\mu)=\frac{1}{2^{m} \bar{\Pi} m}\left(1-\mu^{2}\right)^{\frac{m}{2}} F\left(m-t, m+t+1, m+1, \frac{1-\mu}{2}\right) \ldots
$$

When $t-m$ is 0 or a positive integer $p$, the hypergeometric function here is a rational integral function of $\mu$, and we have

$$
\begin{gathered}
P_{m+p}^{m}(\mu)=\frac{1}{2^{m} \Pi m}\left(1-\mu^{2}\right)^{\frac{m}{2}} \frac{1}{2^{p}} \frac{(2 m+p+1)(2 m+p+2) \ldots(2 m+2 p)}{(m+1)(m+2) \ldots(m+p)} \\
\cdot\left\{\mu^{p}-\frac{p(p-1)}{2(2 m+2 p-1)} \mu^{p-2}+\frac{p(p-1)(p-2)(p-3)}{2.4(2 m+2 p-1)(2 m+2 p-3)} \mu^{p-r}-. \cdot\right\}
\end{gathered}
$$

The integral (1) or the series (2) is convergent if $m>-\frac{1}{2}$, unless one of the three $a, b, c$ or $\alpha, \beta, \gamma$ is equal to the sum of the other two; in the latter case we must have $m>\frac{1}{2}$, and the value of the integral or series is then zero.

The results (1) and (2) admit of specially simple treatment in the symmetrical case $(m=0)$, and we will consider this case first.

2. Let $z, \rho, \phi$ be the cylindrical coordinates of a point. The function

$$
\int_{0}^{\infty} e^{-\lambda z} J_{0} \lambda \rho d \lambda
$$

* $C f$. a paper, “The Determination of Green's Funotion by means of Cylindrical or Spherical Hermonica." Proc. Itdin. Math. Soc., Vol. XVIII., 1899-1900. 
where $z>0$, is a harmonic function which is symmetrical about the axis of $z$. Its value on the axis is

Hence

$$
\int_{0}^{\infty} e^{-\lambda z} d z \text {, or } \frac{1}{z}
$$

$$
\int_{0}^{\infty} e^{-\lambda z} J_{0} \lambda \rho d \lambda=\frac{1}{\sqrt{ }\left(z^{2}+\rho^{2}\right)},
$$

for two symmetrical harmonic functions having the same value on the axis of symmetry are identical.

Again,

$$
\int_{0}^{\infty} e^{-\lambda z} J_{0} \lambda \rho J_{0} \lambda a d \lambda
$$

is a symmetrical harmonic whose value on the axis is

$$
\begin{gathered}
\int_{0}^{\infty} e^{-\lambda z} J_{0} \lambda a d \lambda, \\
\text { i.e. } \frac{1}{\sqrt{ }\left(z^{2}+a^{2}\right)}, \text { by }(6) .
\end{gathered}
$$

This potential on the axis can be produced by unit mass on the plane $z=0$ at distance $a$ from the axis; and it will be a symmetrical potential if the unit mass is distributed uniformly round the axis. Hence

$$
\int_{0}^{\infty} e^{-\lambda z} J_{0} \lambda \rho J_{0} \lambda a d \lambda
$$

is the potential at distance $\rho$ from the axis due to unit mass dis. tributed uniformly over the rim of the circle $(z=0, \rho=a)$.

Next,

$$
\int_{0}^{\infty} e^{-\lambda z} J_{0} \lambda \rho J_{0} \lambda a J_{0} \lambda b d \lambda
$$

is a symmetrical harmonic whose value on the axis is

$$
\int_{0}^{\infty} e^{-\lambda z} J_{0} \lambda a J_{0} \lambda b d \lambda
$$

by the preceding case this value on the axis will be produced by unit mass distributed uniformly over the rim of a circle of radius $b$ with centre distant $a$ from the axis. Take $a \geq b$.

If now any element of this unit mass distant $\rho$ from the axis be distributed uniformly over the circle of radius $\rho$ in the plane $z=0$ with centre at the origin, the potential on the axis will not be changed, and the potential will now be symmetrical. 
Hence we can easily calculate the density of the surface distribution on the plane $z=0$ which will produce the potential given when $z>0$ by

$$
\int_{0}^{\infty} e^{-\lambda z} J_{0} \lambda_{\rho} J_{0} \lambda a J_{0} \lambda b d \lambda
$$

Let $C$ be the centre of the circle of radius $b, O C=a, P$ a point on this circle, $O P=\rho$, angle $O C P=\psi$. The circle of radius $\rho$ about $O$ cuts the circle about $C$ at a second point $P^{\prime}$. The two elements at $P$ and $P^{\prime}$ have together a mass $d \psi / \pi$, which is distributed over an area $2 \pi \rho d \rho$, giving a surface density

$$
\sigma=\frac{1}{2 \pi^{2} \rho} \frac{d \psi}{d \rho}
$$

Now, geometrically,

$$
\begin{aligned}
\frac{d \rho}{b d \psi} & =\sin O P C \\
& =\frac{2 \Delta}{b \rho},
\end{aligned}
$$

where $\Delta$ is the area of the triangle $O P C$.

(Otherwise, since $\rho^{2}=a^{2}+b^{2}-2 a b \cos \psi$, we have

$$
\rho d \rho=a b \sin \psi d \psi \text {.) }
$$

Thus

$$
\begin{aligned}
2 \pi v & =\frac{d \psi}{\pi \rho d \rho} \\
& =\frac{1}{2 \pi \Delta} .
\end{aligned}
$$

But, the potential for $z>0$ being

$$
\int_{0}^{\infty} e^{-\lambda z} J_{0} \lambda \rho J_{0} \lambda a J_{0} \lambda b d \lambda
$$

the value of $2 \pi \sigma$ is $L t\left(-\frac{\partial V}{\partial z}\right)_{z=0}$.

Hence

$$
\int_{0}^{\infty} e^{-\lambda z} J_{0} \lambda \rho J_{0} \lambda a J_{0} \lambda b \lambda d \lambda=\frac{1}{2 \pi \Delta}
$$

which agrees with (1).

3. This method can be extended at once to a spherical surface. Let, $r, \theta, \phi$ be the spherical coordinates of a point.

The function

$$
\sum_{p=0}^{p=\infty} r^{-p-1} P_{p}(\cos \theta)
$$


where $r>1$, is a harmonic function which is symmetrical about the polar axis. Its value on the axis is

$$
\sum_{p=0} r^{-p-1} \text {, i.e. } \frac{1}{r-1},
$$

which is the potential on the axis of unit mass at the point $(r=1, \theta=0)$.

Hence

when $r>1$; and similarly

$$
\sum_{p=0} r^{-p-1} P_{p}(\cos \theta)=\frac{1}{\sqrt{\left(r^{2}-2 r \cos \theta+1\right)}}
$$

when $r<1$.

$$
\sum_{p=0} r^{p} P_{p}(\cos \theta)=\frac{1}{\sqrt{\left(r^{2}-2 r \cos \theta+1\right)}},
$$

Again,

$$
\sum_{p=0} r^{-p-1} P_{p}(\cos \theta) P_{p}(\cos \alpha)
$$

is a symmetrical harmonic function whose value on the axis is

$$
\text { or, by (8), } \quad \begin{aligned}
& \sum_{p=0} r^{-p-1} P_{p}(\cos \alpha), \\
& \frac{1}{\sqrt{\left(r^{2}-2 r \cos \alpha+1\right)}} .
\end{aligned}
$$

This potential on the axis can be produced by unit mass on the sphere $r=1$ at angular distance $\alpha$ from the axis; and it will be a symmetrical potential if the unit mass is distributed uniformly round the axis. Hence

$$
\sum_{p=0} r^{-p-1} P_{p}(\cos \theta) P_{p}(\cos \alpha),(r>1)
$$

is the potential at angular distance $\theta$ from the axis due to unit mass distributed uniformly over the $\operatorname{rim}$ of the circle $(r=1, \theta=\alpha)$. Next,

$$
\sum_{p=0} r^{-p-1} P_{p}(\cos \theta) P_{p}(\cos \alpha) P_{p}(\cos \beta),(r>1)
$$

is a symmetrical harmonic whose value on the axis is

$$
\sum_{p=0} r^{-p-1} P_{p}(\cos \alpha) P_{p}(\cos \beta)
$$

by the preceding case this value on the axis will be produced by unit mass distributed uniformly over the rim of a circle of angular radius $\beta$ with centre at angular distance $\alpha$ from the axis. Take $\alpha \geq \beta$. If, now, any element of this unit mass at angular distance $\theta$ from the axis be distributed uniformly over the rim of the circle 
$(r=1, \theta=\theta)$, the potential will be symmetrical, and its value on the axis will not be changed.

Let $O$ be the point $(r=1, \theta=0), C$ the pole of the circle of radius $\beta, P$ a point on this circle, and, in the spherical triangle $O C P$,

$$
O C=a, C P=\beta, O P=\theta \text {, angle } O C P=\psi .
$$

The circle of radius $\theta$ about $O$ cuts the circle about $C$ at a second point $\mathrm{P}^{\prime}$. The two elements at $P$ and $P^{\prime}$ have a mass $d \psi / \pi$, which is distributed over an area $2 \pi \sin \theta d \theta$, giving the surface density

$$
\sigma=\frac{1}{2 \pi^{2} \sin \theta} \frac{d \psi}{d \theta}
$$

But

so that

$$
\begin{aligned}
\cos \theta & =\cos \alpha \cos \beta+\sin \alpha \sin \beta \cos \psi, \\
\sin \theta d \theta & =\sin \alpha \sin \beta \sin \psi d \psi,
\end{aligned}
$$

$$
\begin{aligned}
& \sigma=\frac{1}{2 \pi^{2} \sin \alpha \sin \beta \sin \psi}
\end{aligned}
$$

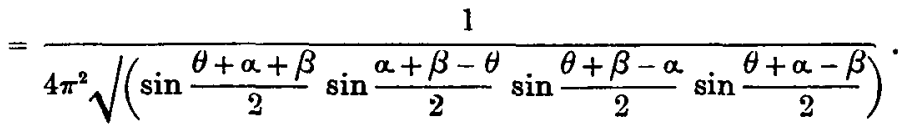

Now, since the potential is

$$
\sum_{p=0} r^{-p-1} P_{p}(\cos \theta) P_{p}(\cos \alpha) P_{p}(\cos \beta),(r>1)
$$

and

we have

$$
\left.\sum_{p=0} r^{p} P_{p}(\cos \theta) P_{p}(\cos \alpha) P_{p}(\cos \beta), r<1\right)
$$

$$
\begin{aligned}
4 \pi \sigma & =\operatorname{Lt}\left(\frac{\partial V}{\partial r}\right)_{r=1-}+\operatorname{Lt}\left(-\frac{\partial V}{\partial r}\right)_{r=1+} \\
& =\sum_{p=0}(2 p+1) P_{p}(\cos \theta) P_{p}(\cos \alpha) P_{p}(\cos \beta)
\end{aligned}
$$

Thus

$$
=\frac{\sum_{p=0}(2 p+1) P_{p}(\cos \theta) P_{p}(\cos \alpha) P_{p}(\cos \beta)}{\pi \sqrt{ }\left(\sin \frac{\theta+\alpha+\beta}{2} \sin \frac{\alpha+\beta-\theta}{2} \sin \frac{\theta+\beta-\alpha}{2} \sin \frac{\theta+\alpha-\beta}{2}\right)} .
$$


or 0 , according as $\alpha, \beta, \theta$ can form the sides of a spherical triangle or not. This agrees with (2).

4. A modification of the argument of the two preceding articles may be noted here, as the modified treatment will be practically obligatory in the asymmetrical case.

The potential

$$
\int_{0}^{\infty} e^{-\lambda z} J_{0} \lambda \rho J_{0} \lambda a d \lambda
$$

being due to unit mass distributed uniformly over the rim of the circle $(z=0, \rho=a)$, we have

$$
\int_{0}^{\infty} e^{-\lambda z} J_{0} \lambda \rho J_{0} \lambda a d \lambda=\frac{1}{2 \pi} \int_{-\pi}^{\pi} \frac{d \psi}{\sqrt{\left(z^{2}+\rho^{2}-2 a \rho \cos \psi+a^{2}\right)}},
$$

Hence the value on the axis of the potential

$$
\int_{0}^{\infty} e^{-\lambda z} J_{0} \lambda_{\rho} J_{0} \lambda a J_{0} \lambda b d \lambda
$$

is

$$
\frac{1}{\pi} \int_{0}^{\pi} \frac{d \psi}{\sqrt{\left(z^{2}+a^{2}-2 a b \cos \psi+b^{2}\right)}} .
$$

Put $a^{2}-2 a b \cos \psi+b^{2}=u^{2}$, and the last integral becomes

$$
\frac{1}{\pi} \int_{a-b}^{a+b} \frac{1}{a b \sin \psi} \frac{u d u}{\sqrt{\left(z^{2}+u^{2}\right)}} \text {. }
$$

But

$$
\int_{c}^{d} \frac{f(u) 2 \pi u d u}{\sqrt{ }\left(z^{2}+u^{2}\right)}
$$

is the potential on the axis due to surface density $f(\rho)$ on the plane $z=0$ from $\rho=c$ to $\rho=d$.

Hence in the present case

$$
f(\rho)=\frac{1}{2 \pi^{2} a b \sin \psi}
$$

from $\rho=a-b$ to $\rho=a+b$, where

$$
a^{2}+b^{2}-2 a b \cos \psi=\rho^{2}
$$

which is the same result as in Art. 2.

5. In order to arrive at the general results (1) and (2) we have to consider harmonic functions of the type

$$
\rho^{m} e^{i m \phi} F(\rho, z) \text {, }
$$


which we will call harmonics of rank $m$. A harmonic of this type, unless $m$ happens to be an integer, requires for its full exhibition a space winding about the axis of $z$, in-which the variable $\phi$ ranges from $-\infty$. to $+\infty$.

If $D$ is the distance from $(\rho, \phi, z)$ to $\left(\rho^{\prime}, \phi^{\prime}, z^{\prime}\right)$, the potential $1 / D$ has a unit singularity of the first order not only at $\left(\rho^{\prime}, \phi^{\prime}, z^{\prime}\right)$, but: also at ' $\left.\rho^{\prime} . \phi^{\prime}+2 k \pi, z^{\prime}\right)$, where $k$ is any integer. The potential having unit singularity of the first order at $\left(\rho^{\prime}, \phi^{\prime}, z^{\prime}\right)$ alone is known, but instead of assuming this knowledge we prefer to investigate independently an expression for an equally fundamental potential which has a linear distribution of singularities of the first order, of linear density $e^{i m \phi} / a$, on the rim of the circle, $(\rho=a, z=0)$.

This potential may be constructed in the following way. Begin with the ordinary physical case of matter distributed with linear density $e^{i m \phi} / a$ from $\phi=\phi_{1}$ to $\phi=\phi_{2}$, where $-\pi<\phi_{1}<\phi_{2}<\pi$. The potential is

where

$$
\begin{gathered}
\int_{\phi_{1}}^{\phi_{2}} \frac{e^{i m \psi} d \psi}{\sqrt{\left\{z^{2}+\rho^{2}-2 a \rho \cos (\psi-\phi)+a^{2}\right\}}} \\
=\frac{1}{\sqrt{(2 a \rho)}} \int_{\phi_{1}}^{\phi_{2}} \frac{e^{i m \psi} d \psi}{\sqrt{ }\{\cosh \alpha-\cos (\psi-\phi)\}},
\end{gathered}
$$

$$
\cosh \alpha=\frac{z^{2}+\rho^{2}+a^{2}}{2 a \rho}
$$

We take $\alpha$ as positive. Since

$$
(\cosh \alpha-1) 2 a \rho=z^{2}+(\rho-a)^{2},
$$

we have $a>0$, except on the circle $(z=0, \rho=a)$.

Put

$$
I=\frac{1}{\sqrt{(2 a \rho)}} \int_{\phi_{1}}^{\phi_{2}} \frac{e^{i m \psi} d \psi}{\sqrt{ }\{\cosh \alpha-\cos (\psi-\phi)\}} .
$$

Now regard $\psi$ as a complex variable, and the integral $I$ as a complex integral taken along the straight path from $\phi_{1}$ to $\phi_{2}$ in the $\psi$ plane. The integrand has branch points where $\psi=2 k \pi+\phi \pm i \alpha$. In the $\psi$ plane draw crosscuts parallel to the imaginary axis, to $+\infty i$ from the branch points with positive imaginary part, and to $-\infty i$ from the branch points with negative imaginary part. Then take

$$
J=\frac{1}{\sqrt{(2 a \rho)}} \int \frac{e^{\imath m \psi} d \psi}{\sqrt{ }\{\cosh \alpha-\cos (\psi-\phi)\}},
$$


the path being from $\phi_{1}+\infty i$ to $\phi_{1}$, then from $\phi_{1}$ to $\phi_{2}$, then from $\phi_{2}$ to $\phi_{2}+\infty i$. The contributions to $J$ from the first and third parts of the path are harmonic functions having no singularity (outside the axis $\rho=0$ ) when $\phi$ lies between $\phi_{1}$ and $\phi_{2}$, even when $\alpha=0$. Thus the harmonic function $J$ has (outside the axis) the same singularity as $I$, when $\alpha=0$ and $\phi$ lies between $\phi_{1}$ and $\phi_{2}$.

But, contracting the path of $J$ until it just skirts the crosscut through $\phi+i \alpha$, we find

$$
J=\frac{2}{\sqrt{ }(2 a \rho)} \int_{a}^{\infty} \frac{e^{i m \phi} e^{-m v} d v}{\sqrt{ }(\cosh v-\cosh \alpha)} .
$$

$J$, being proportional to $e^{i m \phi}$, and having the singularity required when $\phi$ is between $\phi_{1}$ and $\phi_{2}$, has the proper singularity for all values of $\phi$.

In (13) put $e^{-v}=u$.

Thus

$$
\begin{aligned}
& J=\frac{-2}{\sqrt{(a \rho)}} e^{i m \phi} \int_{0}^{e^{-\alpha}} \frac{u^{m-1} d u}{\sqrt{\left(u^{2}-2 u \cosh \alpha+1\right.}} \ldots \\
& \text { In (14) put } \quad u-\sqrt{u^{2}-2 u \cosh \alpha+1}=w,
\end{aligned}
$$

so that

$$
\frac{d u}{u-w}=\frac{d w}{\cosh \alpha-w} \text {, }
$$

and

$$
u=\frac{1-w^{2}}{2(\cosh \alpha-w)} ;
$$

then

$$
J=\frac{2}{\sqrt{ }(a \rho)} e^{i m \phi} \int_{-1}^{e^{-a}} \frac{\left(1-w^{2}\right)^{m-\frac{1}{2}} d w}{2^{m-\frac{1}{2}}(\cosh \alpha-w)^{m+\frac{1}{2}}} .
$$

Similarly, by putting in (14)

we find

$$
u+\sqrt{u^{2}-2 u \cosh \alpha+1}=w,
$$

$$
J=\frac{2}{\sqrt{ }(a \rho)} e^{i m \phi} \int_{e^{-\alpha}}^{1} \frac{\left(1-w^{2}\right)^{m-\frac{1}{2}} d w}{2^{m-1}(\cosh \alpha-w)^{m+\frac{1}{3}}} .
$$

By adding (15) and (16) and putting $w=\cos t$, we obtain

$$
J=\frac{1}{2^{n-\frac{t}{2}} \sqrt{(a \rho)}} e^{i m \phi} \int_{0}^{\pi} \frac{\sin ^{2 m} t d t}{(\cosh \alpha-\cos t)^{m+1}},
$$

or, finally,

$$
J=2 a^{m} \rho^{m} e^{i m \phi} \int_{0}^{\pi} \frac{\sin ^{2 m} t d t}{\left(z^{2}+\rho^{2}-2 a \rho \cos t+a^{2}\right)^{m+\frac{1}{2}}} .
$$


6. We have proved that the function $J$ of (18) is a harmonic function having a linear distribution of singularities of the first order of density $e^{i m \phi} / a$ on the rim of the circle $(\rho=a, z=0)$. $J$ is of the form $\rho^{m} e^{i m \phi} f(\rho, z)$, and

$$
f(0, z)=\frac{2 a^{m}}{\left(z^{2}+a^{2}\right)^{m+\frac{1}{3}}} \cdot \frac{\sqrt{\pi} \Pi\left(m-\frac{1}{2}\right)}{\Pi m}
$$

By integration, a surface distribution of singularities of the first order with surface density $\sigma(\rho) e^{i m \phi}$ from $\rho=p$ to $\rho=q$ on $z=0$ will give a potential $\rho^{m} e^{i m \phi} F(\rho, z)$, where

$$
F(0, z)=\frac{2 \sqrt{\pi} \Pi\left(m-\frac{1}{2}\right)}{\mathrm{II} m} \int_{p}^{a} \frac{u^{m+1} \sigma(u) d u}{\left(z^{2}+u^{2}\right)^{m+\frac{1}{t}}},
$$

Now it can be proved that a harmonic function of rank $m$ say $\rho^{m} e^{i m \phi} F(\rho, z)$ is determined by the value of $F(\rho, z)$ on the axis, just as in the case of a symmetrical harmonic. Hence in any case where $F(0, z)$ can be thrown into the form (20) we can read off at once the value of the surface density on $z=0$.

$$
\text { If } F(0, z)=\int_{p}^{x} \frac{\chi(u) d u}{\left(z^{2}+u^{2}\right)^{m+\frac{1}{z}}},
$$

then

$$
\sigma(\rho)=\frac{\Pi m}{2 \sqrt{\pi} \Pi\left(m-\frac{1}{2}\right)} \frac{\chi(\rho)}{\rho^{m+1}},
$$

from $\rho=p$ to $\rho=q$, and $\sigma(\rho)=0$ outside those limits.

7. We have

$$
\int_{0}^{\infty} e^{-\lambda z} J_{m} \lambda \rho \cdot \lambda^{m} d \lambda=\frac{\Pi(2 m)}{2^{m} \Pi m} \frac{\rho^{m}}{\left(z^{2}+\rho^{2}\right)^{m+\frac{1}{2}}},
$$

for each side, when multiplied by $e^{i m \phi}$, is a harmonic function of rank $m$, taking the form

near $\rho=0$.

$$
\frac{\Pi(2 m)}{2^{m} \bar{\Pi} m} \frac{\rho^{m}}{z^{2 m+1}}
$$

Again,

$$
e^{i m \phi} \int_{0}^{\infty} e^{-\lambda z} J_{m} \lambda \rho J_{m} \lambda a d \lambda
$$


is a harmonic of rank $m$, taking, near $\rho=0$, the form

$$
\frac{\rho^{m} e^{i m \phi}}{2^{m} \Pi m} \int_{0}^{\infty} e^{-\lambda z} J_{m} \lambda a \lambda^{m} d \lambda,
$$

i.e. by (22),

$$
\frac{\Pi(2 m)}{\left(2^{m} \Pi m\right)^{2}} e^{i m \phi} \frac{\rho^{m} a^{m}}{\left(z^{2}+a^{2}\right)^{m+\frac{1}{2}}}
$$

or

since

$$
\frac{\Pi\left(m-\frac{1}{2}\right)}{\sqrt{\pi} \Pi m} e^{i m \phi} \frac{\rho^{m} a^{m}}{\left(z^{2}+a^{2}\right)^{m+\frac{1}{l}}}
$$

$$
\Pi(2 m)=\frac{1}{\sqrt{\pi}} 2^{2 m} \Pi m \Pi\left(m-\frac{1}{2}\right) .
$$

Comparing with (18) and (19) we infer that

$$
\int_{0}^{\infty} e^{-\lambda z} J_{m} \lambda \rho J_{m} \lambda a d \lambda=\frac{1}{\pi} \rho^{m} a^{m} \int_{0}^{\pi} \frac{\sin ^{2 m} t d t}{\left(z^{2}+\rho^{2}-2 a \rho \cos t+a^{2}\right)^{m+\frac{1}{\jmath}}}, \ldots
$$

and that each of the functions of (23) is a harmonic function having a linear distribution of singularities of the first order of linear density $e^{i m \phi} / 2 \pi a$ on the rim of the circle $(\rho=a, z=0)$; briefly, each is the potential due to a ring of sources on the rim of the circle $(\beta=a, z=0)$ of linear density $e^{i m \phi} / 2 \pi a$.

Consider next the harmonic function

$$
e^{i m \phi} \int_{0}^{\infty} e^{-\lambda z} J_{m} \lambda \rho J_{m} \lambda a J_{m} \lambda b d \lambda
$$

The form of this near $\rho=0$ is

$$
\frac{\rho^{m} e^{i m \phi}}{2^{m} \Pi m} \int_{0}^{\infty} e^{-\lambda z} J_{m} \lambda a J_{m} \lambda b d \lambda,
$$

or, by (23),

$$
\frac{\rho^{m} e^{i m \phi}}{2^{m} \Pi m} \cdot \frac{1}{\pi} a^{m} b^{m} \int_{0}^{\pi} \frac{\sin ^{2 m} t d t}{\left(z^{2}+a^{2}-2 a b \cos t+b^{2}\right)^{m+\frac{1}{2}}} .
$$

In (25) put $a^{2}-2 a b \cos t+b^{2}=u^{2}$, and suppose $a \geq b$.

(25) becomes 


$$
\frac{\rho^{m} e^{i m \phi}}{2^{m} \Pi m} \cdot \frac{1}{\pi} a^{m-1} b^{m-1} \int_{a-b}^{a+b} \frac{\sin ^{m-m-1} t \cdot u d u}{\left(z^{2}+u^{2}\right)^{m+1}} .
$$

Comparing with (21), we find

$$
\sigma(\rho)=\frac{\Pi m}{2 \sqrt{\pi} \Pi\left(m-\frac{1}{2}\right)} \frac{1}{2^{m} \Pi m} \cdot \frac{1}{\pi} a^{m-1} b^{m-1} \cdot \frac{1}{\rho^{m}} \sin ^{2 m-1} t,
$$

where $t$ is given by

so that

$$
a^{2}-2 a b \cos t+b^{2}=\rho^{2},
$$

$$
\sin t=\frac{2 \Delta}{a b},
$$

$\Delta$ being the area of the triangle with sides $a, b, c$.

But, from (24)

Hence

$$
2 \pi \sigma(\rho)=\int_{0}^{\infty} J_{m} \lambda \rho J_{m} \lambda a J_{m} \lambda b \cdot \lambda^{-m+1} d \lambda
$$

$$
\begin{aligned}
& \int_{0}^{\infty} J_{m} \lambda \rho J_{m} \lambda a J_{m} \lambda b . \lambda^{-m+1} d \lambda \\
& \quad=\frac{\{(a+b+\rho)(b+\rho-a)(\rho+a-b)(a+b-\rho)\}^{m-1}}{\sqrt{\pi} \Pi\left(m-\frac{1}{2}\right) 2^{3 m-1} a^{m} b^{m} \rho^{m}}, \ldots
\end{aligned}
$$

which is (1).

8. To arrive at the theorem (2) we have only to go through the steps of the process from (18) to (26), using spherical coordinates.

Beginning with (18) take the ring as $\left(z=z^{\prime}, \rho=a\right)$. The potential $J$ becomes

$$
2 a^{m} \rho^{m} e^{i m \phi} \int_{0}^{x} \frac{\sin ^{2 m} t d t}{\left\{\left(z-z^{\prime}\right)^{2}+\rho^{2}-2 a \rho \cos t+a^{2}\right\}^{m+\frac{l}{2}}} .
$$

Put $z=r \cos \theta, z^{\prime}=c \cos \alpha, \rho=r \sin \theta, a=c \sin \alpha$.

Then the potential due to a ring of sources of linear density $e^{i m \phi} / c \sin \alpha$ on the circle $(r=c, \theta=\alpha)$ is

$$
\begin{aligned}
J^{\prime}= & 2 r^{m} c^{m} \sin ^{m} \theta \sin ^{\mu} \alpha e^{i m \phi} \\
& \int_{0}^{\pi} \frac{\sin ^{n m} t d t}{\left\{r^{2}-2 c r\left(\cos \theta \cos \alpha+\sin \theta \sin \alpha \cos t+c^{2}\right\}^{m+1}\right.} .
\end{aligned}
$$


$J^{\prime}$ has the form $r^{m} \sin ^{m} \theta e^{i m \phi} f_{1}(\theta, r)$, where

$$
f_{1}(0, r)=\frac{2 c^{m} \sin ^{m} \alpha}{\left(r^{2}-2 c r \cos \alpha+c^{2}\right)^{m+1}} \frac{\sqrt{\pi} \Pi\left(m-\frac{1}{2}\right)}{\Pi m} .
$$

By integration a surface distribution of singularities of the first order on $r=c$ with surface density $\sigma(\theta) e^{i m \phi}$ from $\theta=\gamma$ to $\theta=\delta$ will give a potential $r^{m} \sin ^{m} \theta e^{i m \phi} F_{1}(\theta, r)$, where

$F_{1}^{\prime}(0, r)=\frac{2 \sqrt{\pi} \Pi\left(m-\frac{1}{2}\right)}{\Pi m} c^{m+2} \int_{\gamma}^{\delta} \frac{\sin ^{m+1} u \sigma(u) d u}{\left(r^{2}-2 c r \cos u+c^{2}\right)^{m+\frac{1}{2}}}$.

If

$$
F_{1}(0, r)=\int_{\gamma}^{\delta} \frac{\chi(u) d u}{\left(r^{2}-2 c r \cos u+c^{2}\right)^{m+\frac{1}{2}}} \text {, }
$$

Then

$$
\sigma(\theta)=\frac{\Pi m}{2 \sqrt{\pi} \Pi\left(m-\frac{1}{2}\right)} \frac{\chi(\theta)}{c^{m+2} \sin ^{m+1} \theta},
$$

from $\theta=\gamma$ to $\theta=\delta$, and $\sigma(\theta)=0$, outside those limits.

9. The function

$$
\frac{\rho^{m} e^{i m \phi}}{\left\{\rho^{2}+(z-c)^{2}\right\}^{m+\frac{1}{2}}}
$$

is a solid spherical harmonic, the centre of which is on the axis of $z$ at $(\rho=0, z=c)$.

Near $\rho=0$, its form is

If $r>c$ this is

$$
\frac{\rho^{m} e^{i m \phi}}{\left\{(z-c)^{2}\right\}^{m+\frac{1}{2}}} .
$$

$$
\frac{\rho^{m} e^{i m \phi}}{(r-c)^{2 m+1}}
$$

or

$$
\frac{\sin ^{m} \theta e^{i m \phi}}{r^{i n+1}} \sum_{p=0}^{p=\infty} \frac{\Pi(2 m+p)}{\Pi(2 m) \Pi p} \frac{c^{p}}{r^{p}} .
$$

Multiplying by $c^{m} \Pi(2 m)$, and attending to (4), we get

$$
\begin{gathered}
\sum_{p=0} \frac{\Pi(2 m+p)}{\Pi p} \frac{c^{m+p}}{r^{m+p+1}} P_{m+p}^{m}(\cos \theta) \\
=\frac{I I(2 m)}{2^{n} \bar{\Pi} m} \frac{c^{m} r^{m} \sin ^{m} \theta}{\left(r^{2}-2 c r \cos \theta+c^{2}\right)^{m+1}} .
\end{gathered}
$$

where $r>c$. If $r<c$, interchange $r$ and $c$. 
Again,

$$
e^{i m \phi} \sum_{p=0} \frac{\Pi(2 m+p)}{\bar{\Pi} p} \frac{c^{m+p}}{r^{m+p+1}} P_{m+p}^{m}(\cos \theta) P_{m+p}^{m}(\cos \alpha),
$$

$(r>c)$, is a harmonic of rank $m$ taking near $\theta=0$ the form

i.e. by $(22)^{\prime}$

$$
e^{i m \phi} \sum_{p=0} \frac{\Pi(2 m+p)}{\Pi p} \frac{c^{m+p}}{r^{m+p+1}} \frac{1}{2^{m} \Pi m} \sin ^{m} \theta P_{m+p}^{m}(\cos \alpha),
$$

$$
e^{i m \phi} \frac{\Pi\left(m-\frac{1}{2}\right)}{\sqrt{\pi} \Pi m} \frac{c^{m} r^{m} \sin ^{m} \alpha \sin ^{m} \theta}{\left(r^{2}-2 c r \cos \alpha+c^{2}\right)^{m+\frac{1}{t}}} .
$$

Comparing with (18)' and (19)', we find

$\sum_{p=0} \frac{\Pi(2 m+p)}{\Pi I p} \frac{c^{m+p}}{r^{m+p+1}} P_{m+p}^{m}(\cos \theta) P_{m+p}^{m}(\cos \alpha)$

$$
=\frac{1}{\pi} c^{m} r^{m} \sin ^{m} \alpha \sin ^{m} \theta
$$

$$
\int_{0}^{\pi} \frac{\sin ^{2 m} t d t}{\left\{r^{2}-2 c r(\cos \theta \cos \alpha+\sin \theta \sin \alpha \cos t)+c^{2}\right\}^{m+1}}
$$

and each, when multiplied by $e^{i m \phi}$, is the potential due to a ring of sources of linear density $e^{i m \phi} / 2 \pi c \sin \alpha$ on the circle $(r=c, \theta=\alpha)$.

Consider next the harmonic function

$$
e^{i m \phi} \sum_{p=0} \frac{\Pi(2 m+p)}{\Pi p} \frac{c^{n+p}}{r^{m+p+1}} P_{m+p}^{m}(\cos \theta) P_{m+p}^{m}(\cos \alpha) P_{m+p}^{m}(\cos \beta),
$$

where $r>c$.

The form of this near $\theta=0$ is

$$
e^{i m \phi} \frac{\sin ^{m} \theta}{2^{m} \Pi m} \sum_{p=0} \frac{\Pi(2 m+p)}{\Pi p} \frac{c^{m+p}}{r^{m+p+1}} P_{m+p}^{m}(\cos \alpha) P_{m+p}^{m}(\cos \beta),
$$

or, by (23)'

$$
\begin{gathered}
e^{i m \phi} \frac{\sin ^{m} \theta}{2^{m} \Pi m} \frac{1}{\pi} c^{m} r^{m} \sin ^{m} \alpha \sin ^{m} \beta \\
\int_{0}^{\pi} \frac{\sin ^{2 m} t d t}{\left\{r^{2}-2 c r(\cos \alpha \cos \beta+\sin \alpha \sin \beta \cos t)+c^{2}\right\}^{m+\frac{1}{2}}} .
\end{gathered}
$$

In (25)' put

$\cos \alpha \cos \beta+\sin \alpha \sin \beta \cos t=\cos u$, and suppose $\alpha \geq \beta$ 
(25)' becomes

$$
e^{i m \phi} \frac{\sin ^{m} \theta}{2^{m} \Pi m} \frac{1}{\pi} c^{m} r^{m} \sin ^{m-1} \alpha \sin ^{m-1} \beta \int_{a-\beta}^{\alpha+\beta} \frac{\sin ^{2 m-1} t \cdot \sin u d u}{\left(r^{2}-2 c r \cos u+c^{2}\right)^{m+\frac{1}{2}}} .
$$

Comparing with (21)', we find

$$
\sigma(\theta)=\frac{\Pi m}{2 \sqrt{\pi} \Pi\left(m-\frac{1}{2}\right)} \cdot \frac{1}{2^{m} \Pi m} \cdot \frac{1}{\pi} c^{m} \sin ^{m-1} \alpha \sin ^{m-1} \beta \cdot \frac{1}{c^{m+2} \sin ^{m} \theta} \sin ^{2 m-1} t,
$$

where $t$ is given by

$$
\cos \alpha \cos \beta+\sin \alpha \sin \beta \cos t=\cos \theta,
$$

so that

$\sin \alpha \sin \beta \sin t=2\left(\sin \frac{\theta+\alpha+\beta}{2} \sin \frac{\alpha+\beta-\theta}{2} \sin \frac{\theta+\beta-\alpha}{2} \sin \frac{\theta+\alpha-\beta}{2}\right)$

and

$$
4 \pi c^{2} \sigma(\theta)=\frac{1}{\sqrt{ } \pi \Pi\left(m-\frac{1}{2}\right)} \frac{2^{m}}{\sin ^{m} \theta \sin ^{m} \alpha \sin ^{m} \beta}(\ldots)^{m-\frac{1}{2}}
$$

But from (24)' and the cognate equation for $r<c$,

$$
4 \pi c^{2} \sigma(\theta)=\sum_{p=0}(2 m+2 p+1) \frac{\Pi(2 m+p)}{\Pi p} P_{m+p}^{m}(\cos \theta) P_{m+p}^{m}(\cos \alpha) P_{m+p}^{m}(\cos \beta) .
$$

Equating the two values of $4 \pi c^{2} \sigma(\theta)$ we find (2).

10. The theorem (3) is a generalization of a known theorem for Zonal Harmonics, which was originally stated as an example in Ferrer's Spherical Harmonics. A historical account of the Zonal Harmonic Theorem, with indications of proofs, is given by Heine.*

The method by which, a good many years ago, I found (3), though complete, is long and far from neat, and $I$ do not give it here. But I have thought it worth while to set down the statement of the result in connection with the main subject of this paper.

*Handbuch der Kugelfunctionen, Zweiter Band, s. 368. 